\title{
Some Remarks on the Ground State of Infinite Systems in Statistical Mechanics
}

\author{
D. RUELle \\ Institut des Hautes Etudes Scientifiques, \\ Bures-sur-Yvette - France \\ Received October 25, 1968
}

\begin{abstract}
We investigate the ground states of infinite quantum lattice systems. It is shown in particular that a positive energy operator is associated with these states.

\section{Introduction}

In a series of recent papers $^{1}$ a new approach has been developped for the study of the equilibrium states of infinite systems in statistical mechanics ${ }^{2}$. Classical and quantum lattice systems, and classical continuous systems of particles with hard cores have been considered; their equilibrium states at temperature $T \neq 0$ have been investigated. The present note describes the zero temperature states, i.e. the ground states, of the same systems.

Many of the results which we shall present have proofs similar to and simpler than already published proofs for the case $T \neq 0$. We shall omit these proofs, and present therefore a list of theorems mostly without proofs. It will be remarked however that our results about the ground state are not special cases of results for $T \neq 0$, and that some of them have in fact no obvious counterpart at $T \neq 0$.
\end{abstract}

\section{Infinite Volume Limit for the Ground State}

It will be convenient to work with quantum lattice systems, but the results obtained in this section extend to classical lattice gases and classical continuous systems of particles with hard cores (see Footnote 1).

We let $\mathscr{H}$ be a complex Hilbert space with finite dimension and $\mathscr{H}_{x}$ a copy of $\mathscr{H}$ at each point $x$ of the "lattice" $\boldsymbol{Z}^{v}$. For finite $\Lambda \subset \boldsymbol{Z}^{v}$, let

$$
\mathscr{H}(\Lambda)=\bigotimes_{x \in \Lambda} \mathscr{H}_{x}
$$

1 See Robinson and Ruelle [8], Gallavotti and Miracle [2], Ruelle [10], Lanford and Robinson [5], Robinson [7], Gallavotti and Miracle [3], LanFORD and RoBINson [6]. A general treatment is also given in a forthcoming book [11].

2 Some of the ideas involved appear already in RUELLE [9] and FISHer [1]. 
and let $\mathfrak{A}_{\Lambda}$ be the algebra of all bounded operators on $\mathscr{H}(\Lambda)$. If $\Lambda \cap \Lambda^{\prime}=\emptyset$ we identify $\mathfrak{U}_{\Lambda}$ with a subalgebra of $\mathfrak{U}_{\Lambda \cup A^{\prime}}$ by $A \rightarrow A \otimes 1$, and let $\mathfrak{A}$ be the norm completion of the union of all $\mathfrak{A}_{\Lambda}$. If $a \in \boldsymbol{Z}^{\nu}$, there is a natural isomorphism $\mathscr{H}_{x} \rightarrow \mathscr{H}_{x+a}$, hence a natural isomorphism $\mathscr{H}(\Lambda)$ $\rightarrow \mathscr{H}(\Lambda+a)$; there is thus a natural isomorphism $\mathfrak{A}_{\Lambda} \rightarrow \mathfrak{A}_{\Lambda+a}$ for each $\Lambda$ and an automorphism $\tau_{a}$ of the $C^{*}$-algebra $\mathfrak{A}$ extending all of these. The $\tau_{a}$ give a representation of $\boldsymbol{Z}^{v}$ in aut $\mathfrak{A}$.

We define now an interaction $\Phi$ as a function of finite subsets of $\boldsymbol{Z}^{\nu}$ such that

(a) $\Phi(\emptyset)=0$,

(b) $\Phi(X)$ is a self-adjoint element of $\mathfrak{A}_{X}$,

(c) $\Phi(X+a)=\tau_{a} \Phi(X)$,

(d) $\|\Phi\|=\sum_{X \ni 0} \frac{\|\Phi(X)\|}{N(X)}<+\infty$.

With respect to the norm given by (d) the interactions form a real Banach space 2 . The Hamiltonian $H_{\Lambda}(\Phi)$ corresponding to the finite region $\Lambda \subset \boldsymbol{Z}^{v}$ is defined by

$$
H_{\Lambda}(\Phi)=\sum_{X \subset A} \Phi(X)
$$

We let $H_{\Lambda}^{0}(\Phi)$ be the lowest eigenvalue of $H_{\Lambda}(\Phi)$ and

$$
E_{\Lambda}(\Phi)=N(\Lambda)^{-1} H_{\Lambda}^{0}(\Phi)
$$

It is readily seen that $E_{\Lambda}($.$) is concave on \mathfrak{B}$ and, if $\Phi, \Psi \in \mathfrak{Z}$,

$$
\left|E_{\Lambda}(\Phi)-E_{\Lambda}(\Psi)\right| \leqq\|\Phi-\Psi\| \text {. }
$$

Theorem 1. If $\Lambda \rightarrow \infty$ in the sense of $\mathrm{VAN} \mathrm{HovE}^{3}$, the following limit exists for every $\Phi \in \mathfrak{B}$

$$
\lim _{\Lambda \rightarrow \infty} E_{\Lambda}(\Phi)=E(\Phi)
$$

The proof may be obtained by standard arguments (see [2] or [11]). Remark. If we define

then

$$
\begin{aligned}
P_{\Lambda}(\Phi) & =N(\Lambda)^{-1} \log \operatorname{Tr}_{\mathscr{H}(\Lambda)} \exp \left[-H_{\Lambda}(\Phi)\right], \\
P(\Phi) & =\lim _{\Lambda \rightarrow \infty} P_{\Lambda}(\Phi),
\end{aligned}
$$

$$
\lim _{T \rightarrow 0}\left[-T P\left(T^{-1} \Phi\right)\right]=E(\Phi)
$$

This follows from the continuity of $T P_{\Lambda}\left(T^{-1} \Phi\right)$ in $\Phi$ uniformly in $T, \Lambda$.

Before stating further results, we introduce a number of definitions.

3 We say that $\Lambda$ tends to infinity in the sense of VAN Hove (and we write $\Lambda \rightarrow \infty)$ if, for every finite $X \subset \boldsymbol{Z}^{\nu}, N(\Lambda)^{-1} N_{X}(\Lambda)$ tends to zero; here $N_{\boldsymbol{X}}(\Lambda)$ is the

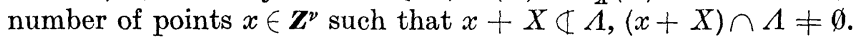


For each $\Phi \in \mathfrak{B}$ we let $V_{\Phi}$ be the subset of the dual $\mathfrak{Z}^{*}$ of $\mathfrak{Z}$ constituted by those elements $\alpha$ such that, for all $\Psi \in \mathfrak{Z}$,

$$
E(\Phi+\Psi) \leqq E(\Phi)+\alpha(\Psi)
$$

The set $V_{\Phi}$ is convex and weakly closed in $\mathfrak{Z}^{*}$. Since $\beta E(\Phi)=E(\beta \Phi)$ for $\beta \geqq 0,(2)$ implies that for all $\Psi$

$$
E(\Psi) \leqq \alpha(\Psi)
$$

hence $V_{\Phi} \subset V_{0}$. From (1) and (3) we get $\|\alpha\| \leqq 1$, therefore (by AlaogluBourbaki) $V_{\Phi}$ is weakly compact. Let $D \subset \mathfrak{Z}$ be the set of those $\Phi$ such that $V_{\Phi}$ is reduced to a point $\alpha^{\Phi}$, i.e. such that the graph of $E$ (.) has a unique tangent plane at $(\Phi, E(\Phi))$. It is known that $D$ is a Baire set ${ }^{4}$ and therefore dense in $\mathfrak{Z}$.

Let I be the set of translationally invariant states on $\mathfrak{A}$, i.e. of those states $\sigma$ such that, for all $a \in \boldsymbol{Z}^{v}, A \in \mathfrak{A}$

$$
\sigma\left(\tau_{a} A\right)=\sigma(A) \text {. }
$$

Given a state $\sigma$ on $\mathfrak{A}$, for each finite $X \subset \boldsymbol{Z}^{v}$ there is a uniquely defined density matrix $\sigma(X)$ such that

$$
\sigma(A)=\operatorname{Tr}_{\mathscr{H}(X)} \sigma(X) A \text { if } A \in \mathfrak{A}_{X} .
$$

The family $(\sigma(X))$ determines $\sigma$. To each $\Phi \in \mathfrak{B}$ we associate $A_{\Phi} \in \mathfrak{A}$ by

$$
A_{\Phi}=\sum_{X \ni 0} \frac{\Phi(X)}{N(X)}
$$

then $\left\|A_{\Phi}\right\| \leqq\|\Phi\|$. To every $\sigma \in I$ there corresponds an element $\omega \sigma$ of $\mathfrak{B}^{*}$

$$
\omega \sigma(\Phi)=\sigma\left(A_{\Phi}\right)=\sum_{X \ni 0} N(X)^{-1} \operatorname{Tr}_{\mathscr{H}(X)} \sigma(X) \Phi(X)
$$

Let $\sigma_{\Lambda}^{\Phi}$ be the orthogonal projection corresponding to the eigenvalue $H_{\Lambda}^{0}(\Phi)$ of $H_{\Lambda}(\Phi)$, divided by the multiplicity of this eigenvalue ${ }^{5}$. The "expectation value in the ground state of $H_{\Lambda}(\Phi)$ " for a (finite) region $X \subset \boldsymbol{Z}^{\nu}$ is defined by a matrix $\sigma_{\Lambda}^{\Phi}(X)$ in $\mathscr{H}(X)$ such that

$$
\sigma_{\Lambda}^{\Phi}(X)=\left\{\begin{array}{lll}
\operatorname{Tr}_{\mathscr{H}(\Lambda X)} \sigma_{\Lambda}^{\Phi} & \text { if } & X \subset \Lambda \\
0 & \text { if } & X \Subset \Lambda .
\end{array}\right.
$$

Averaging over lattice translations we define also

Notice that, if $\Psi \in \mathfrak{Z}$,

$$
\bar{\sigma}_{\Lambda}^{\Phi}(X)=N(\Lambda)^{-1} \sum_{x \in \mathbf{Z}^{\nu}} \sigma_{\Lambda-x}^{\Phi}(X) .
$$

$$
\sum_{X \ni 0} N(X)^{-1} \operatorname{Tr}_{\mathscr{H}(X)} \tilde{\sigma}_{\Lambda}^{\Phi}(X) \Psi(X)=N(\Lambda)^{-1} \operatorname{Tr}_{\mathscr{H}(\Lambda)} \sigma_{\Lambda}^{\Phi} H_{\Lambda}(\Psi)
$$

${ }^{4}$ I.e. $D$ contains a countable intersection of dense open sets in $\mathfrak{B}$.

5 Alternatively, one could define $\sigma_{\Lambda}^{\Phi}$ as the projection on any eigenvector of $H_{\Lambda}(\Phi)$ corresponding to the eigenvalue $H_{\Lambda}^{0}(\Phi)$; this would not change the results described below. 
Using also

we find

$$
\begin{aligned}
N(\Lambda) E_{\Lambda}(\Phi+\Psi) & \leqq \operatorname{Tr}_{\mathscr{H}(\Lambda)} \sigma_{\Lambda}^{\Phi} H_{\Lambda}(\Phi+\Psi) \\
& =N(\Lambda) E_{\Lambda}(\Phi)+\operatorname{Tr}_{\mathscr{H}(\Lambda)} \sigma_{\Lambda}^{\Phi} H_{\Lambda}(\Psi)
\end{aligned}
$$

$$
E_{\Lambda}(\Phi+\Psi) \leqq E_{\Lambda}(\Phi)+\sum_{X \ni 0} N(X)^{-1} \operatorname{Tr}_{\mathscr{H}(X)} \bar{\sigma}_{\Lambda}^{\Phi}(X) \Psi(X) .
$$

Theorem 2. (a) If $\Phi \in D$, then the following limit exists

$$
\lim _{\Lambda \rightarrow \infty} \bar{\sigma}_{\Lambda}^{\Phi}(X)=\sigma^{\Phi}(X)
$$

and the $\sigma^{\Phi}(X)$ are the density matrices associated with a state $\sigma^{\Phi} \in I$ and $\omega \sigma^{\Phi}=\alpha^{\Phi}$.

(b) For all $\Phi \in \mathfrak{Z}$

$$
E(\Phi)=\inf _{\sigma \in I} \sigma\left(A_{\Phi}\right)
$$

and if $\Phi \in D$, then $E(\Phi)=\sigma^{\Phi}\left(A_{\Phi}\right)$.

(c) The mapping $\omega: I \rightarrow 2^{*}$ defined by (4) is an affine homeomorphism of $I$ onto $V_{0}{ }^{6}$. In particular, if $\Phi \in D$, then $\sigma^{\Phi}$ is an extremal point of $I$ ( $Z^{v}$-ergodic state).

The proofs of (a) and (b) follow from standard arguments? ${ }^{7}$. To prove (c), notice first that, since $\omega$ is affine and (weakly) continuous, $\omega I$ is convex and compact. By (b), $\omega I \subset V_{0}$. Furthermore, (b) shows that every closed half-space in $\mathfrak{B}^{*}$ (with the weak topology) which contains all $\omega \sigma^{\Phi}$ (with $\Phi \in D$ ) contains also $V_{0}$, hence $\omega I \supset V_{0}$. Therefore $\omega I=V_{0}$. Since $\omega$ is continuous, and injective by (4), $\omega$ is a homeomorphism ${ }^{8}$.

It is possible in a number of cases to determine explicitly the ground state corresponding to an interaction $\Phi$. By looking at examples of continuous one-dimensional systems of particles with hard cores, the reader will convince himself that $E(\Phi)$ is not in general a piecewise analytic function of the chemical potential.

Remarks. (a) Let $\Phi \in \mathfrak{B}$, from any sequence $\left(\Lambda_{n}\right)$ tending to infinity, one can extract a subsequence $\left(\Lambda_{\alpha}\right)$ such that

$$
\lim _{\alpha \rightarrow \infty} \bar{\sigma}_{\Lambda_{\alpha}}^{\Phi}(X)=\sigma(X)
$$

where the $\sigma(X)$ are the density matrices associated with a state $\sigma \in \omega^{-1} V_{\Phi}$.

${ }^{6}$ This result may be derived from the analysis given in [6]. Conversely, it implies that if $\alpha \in \mathfrak{B}^{*}$ satisfies $\alpha(.) \leqq P($.$) , then \alpha \in \omega I$.

7 See for instance [11]. Notice in fact that, once we know that $E(\Phi) \leqq \sigma\left(A_{\Phi}\right)$ for all $\sigma \in I, \Phi \in \mathfrak{B}$, it suffices to prove (b) for $\Phi \in D$ (because $D$ is dense in $\mathfrak{B}$ and $\|\sigma\|=1$ ). Assuming $\Phi \in D$, (b) follows thus from

$$
\begin{aligned}
\sigma^{\Phi}\left(A_{\Phi}\right) & =\lim _{\Lambda \rightarrow \infty} \sum_{X \ni 0} N(X)^{-1} \operatorname{Tr}_{\mathscr{H}}(\Lambda) \tilde{\sigma}_{\Lambda}^{\Phi}(X) \Phi(X) \\
& =\lim _{\Lambda \rightarrow \infty} N(\Lambda)^{-1} \operatorname{Tr}_{\mathscr{H}}(\Lambda) \sigma_{\Lambda}^{\Phi} H_{\Lambda}(\Phi)=\lim _{\Lambda \rightarrow \infty} N(\Lambda)^{-1} H_{\Lambda}^{0}(\Phi)=E(\Phi) .
\end{aligned}
$$

8 This proof shows in fact that $I$ is the closed convex hull of $\left\{\sigma^{\Phi}: \Phi \in D\right\}$. 
(b) Let $\varrho^{\Phi}$ be a $T=1$ equilibrium state corresponding to $\Phi$ [i.e. $\varrho^{\Phi} \in I$ and $P(\Phi+\Psi) \geqq P(\Phi)-\varrho^{\Phi}\left(A_{\Psi}\right)$ for all $\left.\Psi \in \mathfrak{B}\right]$. If $\Phi \in D$, then

$$
\lim _{T \rightarrow 0} \varrho^{T^{-1} \Phi}=\sigma^{\Phi} .
$$

(c) Let $\mathfrak{B}_{0}$ be the space of finite range interactions, i.e. of those $\Phi \in \mathfrak{Z}$ such that $\Phi(X)=0$ except for finitely many $X \ni 0$. Let also $\mathfrak{B}_{1}$ be any Banach space such that $\mathfrak{B}_{0} \subset \mathfrak{B}_{1} \subset \mathfrak{B}$, the norm \|\|$_{1}$ of $\mathfrak{B}_{1}$ is larger than the norm $\|\cdot\|$ of $\mathfrak{B}$ and $\mathfrak{B}_{0}$ is dense in $\mathfrak{B}_{1}$. Then, $D \cap \mathfrak{B}_{1}$ is a Baire set in $\mathfrak{B}_{1}$ and Theorem 2 remains true with $\mathfrak{B}, \mathfrak{B}^{*}$ replaced by $\mathfrak{B}_{1}, \mathfrak{B}_{1}^{*}$ and $D$ by $D \cap \mathfrak{B}_{1}$.

\section{Time Evolution}

In this section we use in an essential manner the quantum nature of our lattice system, and the results obtained have no interesting counterpart for classical systems.

Following Remark (c) of Section 2, we introduce a Banach space $\mathfrak{B}_{1}$ with

$$
\|\Phi\|_{1}=\sum_{X \ni 0}\|\Phi(X)\| \exp (N(X)-1) .
$$

The following result then holds.

Theorem $3^{9}$. If $\Phi \in \mathfrak{B}_{1}, \mathfrak{A}$ has a one-parameter group of automorphisms $\bar{\tau}: \boldsymbol{R} \rightarrow$ aut $\mathfrak{A}$ such that, if $A \in \mathfrak{A}$,

$$
\bar{\tau}_{t} A=\lim _{\Lambda \rightarrow \infty} e^{\mathrm{it} H_{\Lambda}(\Phi)} A e^{-\mathrm{it} H_{\Lambda^{(\Phi)}}}
$$

uniformly on compact t-intervals; here $A$ tends to infinity in the sense that it eventually contains any finite subset of $\mathbf{Z}^{\nu}$. If $A \in \mathfrak{A}_{X}, X$ finite, the function $t \rightarrow \bar{\tau}_{t} A$ extends to a function $A(z)$ analytic in the strip

$$
\left\{z:|\operatorname{Im} z|<\left(2\|\Phi\|_{1}\right)^{-1}\right\}
$$

with values in $\mathfrak{A}$; for $|z|<\left(2\|\Phi\|_{1}\right)^{-1}$ we have the convergent expansion

$$
A(z)=\sum_{n=0}^{\infty} i^{n} \frac{z^{n}}{n !}\left\{\sum_{X_{1} \subset \mathbf{z}^{\nu}} \cdots \sum_{X_{n} \subset \mathbf{Z}^{\nu}}\left[\Phi\left(X_{n}\right),\left[\cdots\left[\Phi\left(X_{1}\right), A\right] \cdots\right]\right]\right\} .
$$

We can now formulate our main result

Theorem 4. Let $\Phi \in \mathfrak{B}_{1}$ and $\sigma \in \omega^{-1} V_{\Phi}$.

(a) The state $\sigma$ is invariant under time evolution i.e., for all $A \in \mathfrak{A}, t \in \boldsymbol{R}$,

$$
\sigma\left(\bar{\tau}_{t} A\right)=\sigma(A) .
$$

(b) The Gel'fand-Segal construction applied to $\sigma$ yields a Hilbert space $\mathfrak{G}$, a representation $\pi$ of $\mathfrak{A}$ in $\mathfrak{G}$, a normalized vector $\Omega \in \mathfrak{G}$ cyclic with respect to $\pi(\mathfrak{Z})$ and a strongly continuous one-parameter group $t \rightarrow \bar{U}(t)$ of

$\theta$ This is a slightly strengthened version (see [11]) of a theorem due to RoBINson [7], see also [4], [12]. 
unitary operators in $\mathfrak{S}$ such that for all $A \in \mathfrak{A}$,

$$
\begin{aligned}
\sigma(A) & =(\Omega, \pi(A) \Omega), \\
\bar{U}(t) \pi(A) \bar{U}(-t) & =\pi\left(\bar{\tau}_{t} A\right), \\
\bar{U}(t) \Omega & =\Omega .
\end{aligned}
$$

Let $Q$ be the unbounded self-adjoint operator such that

$$
\bar{U}(t)=e^{i Q t}
$$

then the spectrum of $Q$ is contained in $\{q: q \geqq 0\}$.

We prove only the last statement and we assume first that $\Phi \in D$ so that $\sigma=\sigma^{\Phi}$. Let $A, B \in \mathfrak{A}_{X}$. In view of Theorem 2 (a) and Theorem 3 we have, uniformly on compact intervals of $t$ when $\Lambda \rightarrow \infty$ in the sense of VAN Hove,

$$
\begin{aligned}
& \sigma^{\Phi}\left(B^{*} \cdot \bar{\tau}_{t} A\right) \\
& =\lim _{\Lambda \rightarrow \infty} N(\Lambda)^{-1} \sum_{x: x+X \subset \Lambda} \operatorname{Tr}_{\mathscr{H}(\Lambda)} \sigma_{\Lambda}^{\Phi} \tau_{x} B^{*} e^{\mathrm{it} H_{\Lambda}(\Phi)} \tau_{x} A e^{-\mathrm{it} H_{\Lambda}(\Phi)} \\
& =\lim _{\Lambda \rightarrow \infty} N(\Lambda)^{-1} \sum_{x: x+X \subset \Lambda} \operatorname{Tr}_{\mathscr{H}(\Lambda)} \sigma_{\Lambda}^{\Phi} \tau_{x} B^{*} \exp \left[\mathrm{it}\left(H_{\Lambda}(\Phi)-H_{\Lambda}^{0}(\Phi)\right)\right] \tau_{x} A .
\end{aligned}
$$

Therefore, if $\varphi$ is a smooth function with compact support,

$$
\begin{aligned}
& (\pi(B) \Omega, \varphi(Q) \pi(A) \Omega) \\
& \quad=\lim _{\Lambda \rightarrow \infty} N(\Lambda)^{-1} \sum_{x: x+X \subset \Lambda} \operatorname{Tr}_{\mathscr{H}(\Lambda)} \sigma_{\Lambda}^{\Phi} \tau_{x} B^{*} \varphi\left[H_{\Lambda}(\Phi)-H_{\Lambda}^{0}(\Phi)\right] \tau_{x} A .
\end{aligned}
$$

In particular, if the support of $\phi$ is contained in $\{q: q<0\}$, then $\varphi(Q)=0$, proving that the spectrum of $Q$ is contained in $\{q: q \geqq 0\}$. The extension of this result to all $\Phi \in \mathfrak{V}_{1}$ and $\sigma \in \omega^{-1} V_{\Phi}$ can be obtained by a technique described in [6].

Remark. In view of Theorem 4, the ground state expectation values have a number of properties in common with the vacuum expectation values in relativistic quantum field theory. In particular, the function $f\left(\xi_{1}, \ldots, \xi_{n}\right)$ defined by

$$
f\left(t_{1}, t_{2}-t_{1}, \ldots, t_{n}-t_{n-1}\right)=\sigma\left(A_{0} \bar{\tau}_{t_{1}} A_{1} \ldots \bar{\tau}_{t_{n}} A_{n}\right)
$$

is the boundary value of a function $F\left(\zeta_{1}, \ldots, \zeta_{n}\right)$ analytic in

$$
\prod_{i=1}^{n}\left\{\zeta_{i}: \operatorname{Im} \zeta_{i}>0\right\}
$$

\section{Bibliography}

1. Frsher, M. E.: J. Math. Phys. 6, 1643-1653 (1965).

2. Gallavotti, G., and S. Miracle: Commun. Math. Phys. 5, 317-323 (1967).

3. - - Ann. Inst. Henri Poincaré 8, 287-299 (1968).

4. Guenin, M.: Commun. Math. Phys. 3, 120-132 (1966). 
5. Lanford, O., and D. W. Robinson: J. Math. Phys. 9, 1120-1125 (1968).

6. - - Commun. math. Phys. 9, 327-338 (1968).

7. Robinson, D. W.: Commun. Math. Phys. 6, 151-160 (1967); 7, 337-348 (1968).

8. - and D. Ruelle: Commun. Math. Phys. 5, 288-300 (1967).

9. Ruelle, D.: J. Math. Phys. 6, 201-220 (1965).

10. - Commun. Math. Phys. 5, 324-329 (1967).

11. - Statistical mechanics (Rigorous results). New York: Benjamin (to appear).

12. Streater, R. F.: Commun. Math. Phys. 6, 233-247 (1967).

D. Ruelle

Institut des Hautes

Etudes Scientifiques

F 91 Bures-sur-Yvette 